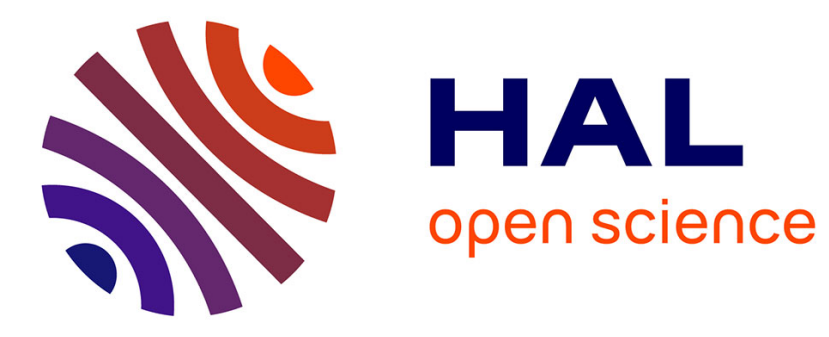

\title{
Critical appraisal of STAT3 pattern in adult cardiomyocytes
}

Zeina Harhous, Sally Badawi, Noelle Gallo Bona, Bruno Pillot, Lionel Augeul, Melanie Paillard, George W Booz, Emmanuelle Canet-Soulas, Michel Ovize, Mazen Kurdi, et al.

\section{To cite this version:}

Zeina Harhous, Sally Badawi, Noelle Gallo Bona, Bruno Pillot, Lionel Augeul, et al.. Critical appraisal of STAT3 pattern in adult cardiomyocytes. Journal of Molecular and Cellular Cardiology, 2019, 131, pp.91-100. 10.1016/j.yjmcc.2019.04.021 . hal-03060376

\section{HAL Id: hal-03060376 https://hal.science/hal-03060376}

Submitted on 14 Dec 2020

HAL is a multi-disciplinary open access archive for the deposit and dissemination of scientific research documents, whether they are published or not. The documents may come from teaching and research institutions in France or abroad, or from public or private research centers.
L'archive ouverte pluridisciplinaire HAL, est destinée au dépôt et à la diffusion de documents scientifiques de niveau recherche, publiés ou non, émanant des établissements d'enseignement et de recherche français ou étrangers, des laboratoires publics ou privés. 


\section{Accepted Manuscript}

Critical appraisal of STAT3 pattern in adult cardiomyocytes

Zeina Harhous, Sally Badawi, Noelle Gallo Bona, Bruno Pillot, Lionel Augeul, Melanie Paillard, George W. Booz, Emmanuelle Canet-Soulas, Michel Ovize, Mazen Kurdi, Gabriel Bidaux

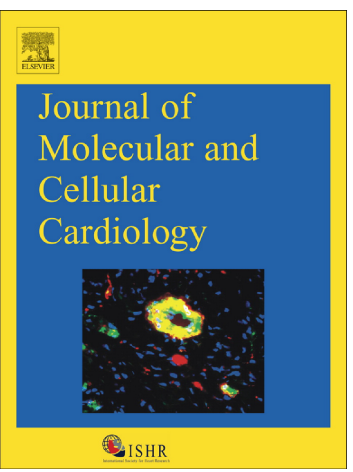

PII: $\quad$ S0022-2828(19)30076-8

DOI: $\quad$ https://doi.org/10.1016/j.yjmcc.2019.04.021

Reference: $\quad$ YJMCC 8976

To appear in: $\quad$ Journal of Molecular and Cellular Cardiology

Received date: $\quad 5$ February 2019

Revised date: $\quad 8$ April 2019

Accepted date: $\quad 19$ April 2019

Please cite this article as: Z. Harhous, S. Badawi, N.G. Bona, et al., Critical appraisal of STAT3 pattern in adult cardiomyocytes, Journal of Molecular and Cellular Cardiology, https://doi.org/10.1016/j.yjmcc.2019.04.021

This is a PDF file of an unedited manuscript that has been accepted for publication. As a service to our customers we are providing this early version of the manuscript. The manuscript will undergo copyediting, typesetting, and review of the resulting proof before it is published in its final form. Please note that during the production process errors may be discovered which could affect the content, and all legal disclaimers that apply to the journal pertain. 


\section{Critical Appraisal of STAT3 Pattern in Adult Cardiomyocytes}

Zeina Harhous ${ }^{1,2,3}$, Sally Badawi ${ }^{1,2,3}$, Noelle Gallo Bona ${ }^{1,2}$, Bruno Pillot ${ }^{1,2}$, Lionel Augeul ${ }^{1,2}$, Melanie Paillard $^{1,2}$, George W Booz ${ }^{4}$, Emmanuelle Canet-Soulas ${ }^{1,2}$, Michel Ovize ${ }^{1,2}$, Mazen Kurdi ${ }^{3, * *}$ mkurdi@ul.edu.lb, Gabriel Bidaux ${ }^{1,2, *}$ gabriel.bidaux@univ-lyon1.fr

${ }^{1}$ Univ-Lyon, CarMeN Laboratory, INSERM 1060, INRA 1397, University Claude Bernard Lyon1, INSA Lyon, Oullins, France.

${ }_{2}^{2}$ IHU OPeRa, Groupement Hospitalier EST, Bâtiment B13, 59 boulevard Pinel, F-69500 Bron, France ${ }^{3}$ Lebanese University, Faculty of Sciences, Doctoral School of Sciences and Technology, Laboratory of Experimental and Clinical Pharmacology, Hadat, Lebanon.

${ }^{4}$ Department of Pharmacology and Toxicology, School of Medicine, The University of Mississippi Medical Center, Jackson, MS USA

*Correspondence to: Dr Gabriel Bidaux: CarMeN Laboratory, IHU Opera, INSERM U1060, Groupement Hospitalier EST, Bâtiment B13, 59 boulevard Pinel, F-69500 Bron, France.

${ }^{* *}$ Correspondence to: Dr Mazen Kurdi: Laboratory of Experimental and Clinical Pharmacology, Lebanese University, Faculty of Sciences, Hadat, Lebanon. 


\section{ABSTRACT}

The signal transducer and activator of transcription 3, STAT3, transfers cellular signals from the plasma membrane to the nucleus, acting as a signalling molecule and a transcription factor. Reports proposed an additional non-canonical role of STAT3 that could regulate the activity of complexes I and II of the electron transport chain and the opening of the mitochondrial permeability transition pore (PTP) after ischemiareperfusion in various cell types. The native expression of STAT3 in heart mitochondria, together with a direct versus an indirect transcriptional role in mitochondrial functions, have been recently questioned.

The objective of the present study was to investigate the cellular distribution of STAT3 in mouse adult cardiomyocytes under basal and stress conditions, along with assessing its presence and activity in cardiac mitochondria using structural and functional approaches. The analysis of the spatial distribution of STAT3 signal in the cardiomyocytes interestingly showed that it is transversely distributed in along the T-tubules and in nucleus. This distribution was neither affected by hypoxia nor by hypoxia/re-oxygenation conditions. Focusing on the mitochondrial STAT3 localization, our results suggest that serine-phosphorylated STAT3 (PS727-STAT3) and total STAT3 are detected in crude but not in pure mitochondria of mouse adult cardiomyocytes, under basal and ischemia-reperfusion conditions. The inhibition of STAT3, with a prevalidated non-toxic Stattic dose, had no significant effects on mitochondrial respiration, but a weak effect on the calcium retention capacity. Overall, our results exclusively reveal a unique cellular distribution of STAT3 in mouse adult cardiomyocytes, along the T-tubules and in nucleus, under different conditions. They also challenge the expression and activity of STAT3 in mitochondria of these cells under basal conditions and following ischemia-reperfusion. In addition, our results underline technical methods, complemental to cell fractionation, to evaluate STAT3 roles during hypoxia-reoxygenation and at the interface between nucleus and endoplasmic reticulum.

Keywords: mitochondria, cardiomyocytes, ischemia-reperfusion, t-tubules, subcellular distribution, mitochondria associated membranes 


\section{INTRODUCTION}

The signal transducer and activator of transcription 3 (STAT3) is a member of the STAT family of proteins. It transfers information from the plasma membrane to the nucleus, acting as a signaling molecule and a transcription factor [1]. It is activated by growth factors, cytokines, inflammation, stress conditions and oncogenic kinases [2]. The activation of STAT3 occurs through phosphorylation of its tyrosine 705 (Y705) residue. PY705-STAT3 molecules form dimers which translocate to the nucleus, bind to certain targeted DNA sequences and regulate expression of various genes [3]. STAT3 is also phosphorylated at its serine 727 (S727) residue. Phosphorylation of S727 has been described to be required and self-sufficient for STAT3 transcriptional activity [4]-[6]. Alternatively, a non-canonical role of STAT3 has been reported in the mitochondria [3], [7]. Mitochondria are major players in ischemia-reperfusion-induced cell death, including through opening of the mitochondrial permeability transition pore [8]-[10]. STAT3 has been suggested to enhance the activity of the electron transport chain, to increase ATP production and to decrease ROS generation by interacting with mitochondria complexes I and II [7], [11]. Recent reports using pharmacological or genetic modulation of STAT3 activity and expression in adult cardiomyocytes and various cell lines propose that mitochondrial STAT3 could contribute to protection against ischemiareperfusion injury by ischemic conditioning [7], [12]-[15]. Alternatively, several attempts and approaches to detect STAT3 in the mitochondria have been unsuccessful, and the specificity of its pharmacological inhibitor, Stattic, raises questions. Besides from the controversial mitochondrial STAT3 localization and roles, an evolving role for STAT3 in the endoplasmic reticulum have been recently discovered, where STAT3 was suggested to contribute to the regulation of calcium homeostasis and apoptosis [16], [17].

Therefore, the aim of the present study was to investigate the cellular distribution of STAT3 in mouse adult cardiomyocytes under basal and stress conditions, in addition to the examination of its expression and activity in mitochondria of these cells under different conditions. Our results mainly show a unique pattern of STAT3 distribution along the T-tubules and in the nucleus, under basal and stress conditions. Besides, using several independent methods, we could confirm neither total nor phosphorylated STAT3 expression in cardiac mouse mitochondria under both baseline and ischemia-reperfusion conditions. Moreover, we were unable to confirm that STAT3 inhibition, by a non-toxic concentration of Stattic, could impair mitochondrial respiration. We finally discuss how STAT3 might, however, play a role in ischemia-reperfusion injury and possible important methodological drawbacks in this field of research. 


\section{MATERIALS AND METHODS}

\subsection{Animals}

The present study was performed following the approval by the local institutional animal research committee ( ${ }^{\circ}$ DR2018-35v2). It conforms to the NIH Guide on the Use of Laboratory Animals (NIH Publication No. 85-23, revised 1996). C57bl/6J male mice aged between 8-12 weeks were obtained from Charles River Laboratories.

\subsection{In vivo Myocardial Ischemia-Reperfusion Injury}

As previously described [18], mice received a sub-cutaneous injection of the anesthetics mix (buprenorphine $0.075 \mathrm{mg} / \mathrm{kg}+$ medetomidine $0.3 \mathrm{mg} / \mathrm{kg}$ ) and the analgesics mix (lidocaine $2 \mathrm{mg} / \mathrm{kg}+$ Alfaxan $80 \mathrm{mg} / \mathrm{kg}$ ). They were then placed on a blanket at $37^{\circ} \mathrm{C}$ until fully asleep. ECG and heart rate were monitored using EGC electrodes, and an oximeter was used for $\mathrm{SpO} 2$ monitoring. A tracheotomy was performed and mice were mechanically ventilated. The hearts was exposed via a left thoracotomy, and myocardial infarction was induced by placing an 8-0 suture around the left anterior descending (LAD) artery. Following $45 \mathrm{~min}$ ischemia, the infarcted area was reperfused for $15 \mathrm{~min}$ through the loosening of the suture. The area at risk were identified by the absence of blue staining and was collected for pure mitochondrial isolation prior to western blot analysis.

\subsection{Cell Culture}

The rat derived H9C2 cell line of cardiomyoblasts and mouse embryonic fibroblasts (MEFs), wild type (WT) and STAT3 knockout (KO), were cultured in Dulbecco's Modified Eagle's Medium (DMEM) (41965-039 Gibco), supplemented with $10 \%$ Fetal Bovine Serum and $100 \mathrm{U} / \mathrm{ml}$ penicillin/streptomycin under a humidified incubator aerated with $5 \% \mathrm{CO}_{2}$ at $37^{\circ} \mathrm{C}$. WT and $\mathrm{KO} \mathrm{MEFs}$ were generated by Valeria Poli (University of Torino, Torino, Italy).

\subsection{Mitochondrial Isolation}

To optimize the yield of intact mitochondria, all procedures were carried out on ice, with the centrifugations performed at $4^{\circ} \mathrm{C}$. Crude mitochondria were used for the assessment of mitochondrial functions, while pure mitochondria were used for the qualitative detection of STAT3 under various conditions.

\subsubsection{Crude Mitochondrial isolation}

As previously described [19], after euthanizing the mice by cervical dislocation, the hearts were extracted while still beating and immediately placed in cold buffer A [70mM sucrose, $210 \mathrm{mM}$ mannitol, 1 $\mathrm{mM}$ EDTA and $50 \mathrm{mM}$ Tris $\mathrm{HCl}(\mathrm{pH}$ 7.4)]. Atria were then removed, and ventricular tissues thoroughly minced with scissors. A Potter-Elvehjem (glass-glass) tissue grinder was used for homogenization. The homogenates were centrifuged at 1,300g for $3 \mathrm{~min}$ and the supernatants then collected and centrifuged at $10,000 \mathrm{~g}$ for $10 \mathrm{~min}$. The mitochondrial pellets were suspended in isolation buffer B (same as buffer A except 
for EDTA $0.1 \mathrm{mM}$ ). The suspensions were centrifuged at $10,000 \mathrm{~g}$ for $10 \mathrm{~min}$ and homogenized in $100 \mu \mathrm{L}$ of buffer B. Protein contents were assessed using Bradford reagent with bovine serum albumin as standard.

\subsubsection{Pure Mitochondrial isolation}

As previously described [20], after euthanizing the mice by cervical dislocation, the hearts were extracted and immediately placed in Isolation Buffer, IB1, (225 mM mannitol, $75 \mathrm{mM}$ sucrose, 0.5\% BSA, $0.5 \mathrm{mM}$ EGTA and $30 \mathrm{mM}$ Tris $\mathrm{HCl} \mathrm{pH} 7.4$ ). Atria were then removed, and the tissues finely minced with scissors. The pieces were then homogenized by applying 10-20 strokes in a glass-teflon tissue grinder. The homogenates were centrifuged at $740 \mathrm{~g}$ for $5 \mathrm{~min}$, and the supernatants were then taken and similarly centrifuged. The new supernatants were collected and centrifuged at $9,000 \mathrm{~g}$ for $10 \mathrm{~min}$ at $4^{\circ} \mathrm{C}$. The pellets were gently resuspended in a Stop Buffer, SB, (225 mM mannitol, $75 \mathrm{mM}$ sucrose, $30 \mathrm{mM}$ Tris-HCl pH7.4) and centrifuged at 10,000g for $10 \mathrm{~min}$. The pellets of crude mitochondria were resuspended in Mitochondria Resuspending Buffer, MRB, (250 mM mannitol, 5 mM HEPES (pH7.4) and 0.5 mM EGTA). For obtaining pure mitochondria, suspensions of crude mitochondrial fractions were layered on top of Percoll medium (225 $\mathrm{mM}$ mannitol, $25 \mathrm{mM}$ HEPES, $1 \mathrm{mM}$ EGTA and 30\% Percoll (vol/vol) in $14 \mathrm{ml}$ thin-wall ultracentrifuge tubes. MRB was finally layered on top of the mitochondrial suspensions to fill up the tubes. The latter were centrifuged at 95,000g for $30 \mathrm{~min}$. Purified mitochondria, localized at the bottom of the tube as a dense band, were collected and washed twice by centrifugation at $6,300 \mathrm{~g}$ for $10 \mathrm{~min}$.

\subsection{Mitochondrial Oxidative Phosphorylation}

Oxidative phosphorylation of $350 \mu \mathrm{g}$ crude mitochondria was measured at $25^{\circ} \mathrm{C}$ using a Clark-type electrode (Oroboros oxygraph, Austria) in $2 \mathrm{~mL}$ of respiration buffer (pH 7.4) [100 mM KCl, $50 \mathrm{mM}$ MOPS,

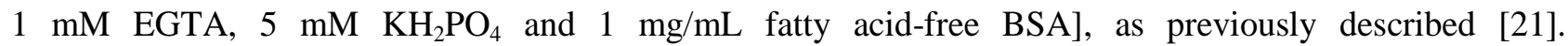
Glutamate/Malate/Pyruvate $(5 \mathrm{mM}$ each), succinate $(5 \mathrm{mM})$ and TMPD-ascorbate $(0.125-1.25 \mathrm{mM}$ respectively) were used as substrates for complexes I, II, and IV respectively. Rotenone $(1 \mu \mathrm{M})$ and Antimicyn A (12.5 $\mu \mathrm{M})$ were respectively added as specific inhibitors of complexes I and III. The maximal oxygen uptake after uncoupling of respiratory chain with FCCP $(2.8 \mu \mathrm{M})$ was also determined. The ADP $(200 \mu \mathrm{M})$-stimulated respiration was measured. The oxygen consumption of the complexes is expressed in nmol $\mathrm{O}_{2} / \mathrm{min} / \mathrm{mg}$ of proteins.

\subsection{Mitochondrial Calcium Retention Capacity (CRC)}

The calcium retention capacity of $250 \mu \mathrm{g}$ of crude mitochondrial proteins was determined in $2 \mathrm{~mL}$ of incubation buffer $\mathrm{C}\left(150 \mathrm{mM}\right.$ sucrose, $50 \mathrm{mM} \mathrm{KCl}, 2 \mathrm{mM} \mathrm{KH_{2 }} \mathrm{PO}_{4}, 20 \mathrm{mM}$ tris/ $\left.\mathrm{HCl}\right)$ at $25^{\circ} \mathrm{C}$ using $5 \mathrm{mM}$ (Glutamate/Malate/Succinate), with ADP-stimulated respiration in the presence of $\mathrm{MgCl}_{2}(1.2 \mathrm{mM})$, as previously described [19]. The extra-mitochondrial $\mathrm{Ca}^{2+}$ concentration was estimated with a spectrofluorometer using $1 \mu \mathrm{M}$ of the calcium sensitive probe calcium green $5 \mathrm{~N}$ (Life technology) (excitation wavelength: 500nm; emission: 530nm). Mitochondria were gently stirred, and $40 \mathrm{nmol} \mathrm{CaCl}_{2}$ pulses were applied every 2 min until a rapid calcium release occurred indicating PTP opening. CRCs were 
expressed in nmoles of $\mathrm{Ca}^{2+} / \mathrm{mg}$ of proteins.

\subsection{Pharmacologic inhibition of STAT3}

A dose-effect assessment of the STAT3 inhibitor, Stattic (sigma-S7946), was tested on isolated mouse cardiomyocytes as follows: cells were treated for 1 or 2 hours, at $37^{\circ} \mathrm{C}$, with either Stattic $(10-100 \mu \mathrm{M})$ or DMSO vehicle. Crude mitochondria were incubated with $50 \mu \mathrm{M}$ Stattic, a concentration which induced a significant decrease in Y705-STAT3 phosphorylation, or with DMSO vehicle for 1 hour at $4^{\circ} \mathrm{C}$. The mitochondrial calcium retention capacities and oxygen consumption were consequently measured.

\subsection{Western blotting}

Proteins were extracted from cells and mitochondria (pure and crude) using complete RIPA lysis buffer with anti-proteases (Sigma; P8340) and anti-phosphatases (Sigma; p5726). The collected proteins were then quantified, and SDS-PAGE Western blotting was performed. Membranes were cut in strips in order to detect several proteins (STAT3 and markers of compartments) at the same time. Reblotting was performed in order to assess the detection of the different forms of STAT3 and different markers of the same size. Membrane strips were blocked for 1 hour with 5\% bovine serum albumin (BSA)-PBS. Immunoblotting was performed by incubating the membranes, overnight at $4^{\circ} \mathrm{C}$, with primary antibodies: Rabbit anti-PY705STAT3 (dil: 1/1,000; Cell Signaling; 9145), rabbit anti-total STAT3 (dil: 1/1,000; Cell Signaling; 4904), mouse anti-PS727-STAT3 (dil: 1/1,000; Santa Cruz; sc-136193) batch 1 (May 2016) and batch 2 (March 2018), mouse anti-GRIM19 (1/200; Santa Cruz; sc- 514111), mouse anti-TOM20 (dil: 1/200; Santa Cruz; sc17764), mouse anti-tubulin (dil: 1/2,000; Santa Cruz; sc-5286), rabbit anti-GAPDH (dil: 1/500; Santa Cruz; sc-25778), mouse anti-cytochrome C (dil: 1/500; Santa Cruz; sc-13156) or anti-NDUFB6 (dil: 1/1,000; Abcam; ms108) prepared in 5\% BSA-PBS. Corresponding horseradish-peroxidase conjugated anti-mouse and anti-rabbit secondary antibodies (dil: 1/10,000) were added and followed by $1 \mathrm{~h}$ incubation at room temperature. Revelation was done using the reaction substrate ECL prime reagent (GE healthcare), and the acquisition was performed with Bio-Rad Molecular Imager Gel Doc XR+ (Bio-Rad). Image Lab software was used for quantification.

\subsection{Hypoxia-Reoxygenation of cardiomyocytes}

Cardiomyocytes were isolated from hearts of $\mathrm{C} 57 \mathrm{bl} / 6 \mathrm{~J}$ mice using Langendorff system, as previously described [22]. They were deposited in 8-well plates and were subjected to 90 min hypoxia ( $0.5 \%$ oxygen) in glucose-free medium (mM: $140 \mathrm{NaCl}, 5 \mathrm{KCl}, 1 \mathrm{MgCl}_{2}, 10 \mathrm{HEPES}$ [pH 7.4], $10 \mathrm{CaCl}_{2}$ ) in a hypoxic incubator (Eppendorf; galaxy 48R-CO48EN200259), followed by $1 \mathrm{~h}$ of reoxygenation (glucose-containing medium). Cells were then immuno-labeled and visualized by microscopy.

\subsection{Immunofluorescence}

Cardiomyocytes, plated in glass Labtech plates (PEZGS0816, Merck Millipore), were fixed for 15 min 
with $2 \%$ paraformaldehyde-PBS prior to 4 washes with PBS. They were then permeabilized with $0.1 \%$ Triton-PBS for 10 min. Following 4 times PBS washes, the nonspecific binding was blocked using 5\% BSAPBS for $1 \mathrm{~h}$ at room temperature (RT). The cells were then incubated for $1 \mathrm{~h}$ at room temperature with primary antibodies prepared in the blocking buffer. Following the primary antibody incubation, the wells were washed 4 times with PBS and corresponding secondary antibodies (Alexa Fluor 488 rabbit and/or Alexa Fluor 647 mouse - 1/2,000 - Jackson Immunoresearch) were applied for another $1 \mathrm{~h}$ at room temperature in darkness. The nuclei were stained through a $10 \mathrm{~min}$ incubation with Bis-benzimide $(5 \mu \mathrm{g} / \mathrm{mL})$. After washing 3 times with PBS, the cells were mounted with Fluoromount ${ }^{\mathrm{TM}}$ Aqueous Mounting Medium (Sigma-F4680) and were gently covered by glass coverslips.

The primary antibodies were: mouse anti-ryanodine receptor (RyR) (Abcam; ab2827) (1/500), mouse anti-PS727-STAT3 (batch 1) (Santa Cruz; sc-136193), rabbit anti-PS727-STAT3 (Cell Signaling; 9134), rabbit anti-total STAT3 (Cell Signaling; 4904), mouse anti-total STAT3 (Santa Cruz; sc-8019), rabbit antiTOM22 (Santa Cruz, sc-14896) and mouse anti-GRIM19 (Santa Cruz; sc- 514111) all diluted at 1/200.

\subsection{Cloning of STAT3-eYFP}

Mouse STAT3 coding sequence was amplified by PCR with High Fidelity Phusion DNA polymerase (Finnzymes; Thermo Fisher Scientific, Waltham, MA). After a $0.8 \%$ (w/vol) agarose-gel extraction of specific DNA bands (Wizard SV gel and PCR Clean-Up System; Promega, Madison, WI), PCR products and recipient vector pVLL-42-mEYFP [23] were digested with High Fidelity NheI and AgeI (New England Biolabs, Ipswich, MA) at $37^{\circ} \mathrm{C}$ overnight, cleaned-up on column prior to be ligated with $\mathrm{T} 4$ ligase (New England Biolabs) at $16^{\circ} \mathrm{C}$ overnight. Products were transformed in JM109 chemo-competent bacteria (New England Biolabs). Plasmid clones were extracted and sequenced before experiments.

\subsection{Mass Spectrometry}

The sample was analyzed using an Ultimate 3000 nano-RSLC (Thermo Scientific, San Jose California) coupled on line with a $Q$ Exactive HF mass spectrometer via a nano-electrospray ionization source (Thermo Scientific, San Jose California). Proteins were finally identified by database searching using SequestHT with Proteome Discoverer 2.2 software (Thermo Scientific) against the swissprot Mus musculus database. Precursor mass tolerance was set at $10 \mathrm{ppm}$ and fragment mass tolerance was set at $0.02 \mathrm{Da}$, and up to 2 missed cleavages were allowed. Oxidation (M), acetylation (Protein N-terminus) were set as variable modification, and Carbamidomethylation $(\mathrm{C})$ as fixed modification. Proteins were filtered with a $1 \%$ false discovery rate.

\subsection{Confocal Imaging}

Confocal imaging was performed using a confocal microscope (Nikon Eclipse Ti, A1R confocal microscope) with a 40x oil immersion objective (N.A. 1.3), equipped with thermos-controlled chamber. The laser lines 405, 488, 514 and $633 \mathrm{~nm}$ were used for excitation. The emission wavelengths were acquired sequentially to prevent spectral overlapping. The depth of the focal plan was $0.5 \mu \mathrm{m}$, pixel size was chosen 
between $60 \mathrm{~nm}$ to $90 \mathrm{~nm}$ in order to respect the Shannon criterion and images were acquired by averaging 4 scanning-lines. Images were further processed with NIS software (Nikon) to adjust brightness and contrast and to calculate the spatial cross-correlation of the two fluorescent signals.

\subsection{Statistics}

All values are expressed as mean \pm standard error of the mean (SEM) for $n$ number of independent observations. Data were analyzed with GraphPad Prism 5.0 using one way ANOVA followed by Dunnett's multiple comparison test or using $t$-test. $P<0.05$ was considered indicative of a significant difference. 


\section{RESULTS}

\subsection{STAT3 is detected in crude but not pure heart, liver and brain mitochondria}

We focused on crude and pure mitochondrial protein extracts by western blotting. In crude cardiac mitochondrial fraction, phosphorylated (PY705 and PS727) and total STAT3 forms were detected, along with the mitochondrial proteins GRIM-19 and Tom20. However, the cytoplasmic protein tubulin was also strongly detected, which indicates fraction contamination with cytosolic proteins $\underline{(n=3)}$ (Fig. 1A). Specificity of anti PS727-STAT3 for phosphate group was assessed by a serine phosphatase treatment that gradually removed the signal (Fig. S1). Since the crude mitochondrial fraction is not reliable for true assessment of exact STAT3 localization, we proceeded toward enriched mitochondrial investigation. Under baseline aerobic conditions, the western blot analysis of cytosolic and enriched, usually defined as "pure", mitochondrial fractions extracted from heart, liver, and brains $\underline{(n=3)}$ of C57bl6J mice showed that PS727STAT3 and total STAT3 were exclusively expressed in the cytosolic fraction, along with the cytosolic proteins tubulin and GAPDH (Fig. 1B). On the pure mitochondrial level, the mitochondrial protein markers NDUFB6 (complex I subunit) and GRIM-19 were detected, ensuring the validity of this fraction.

During ischemia, STAT3 is activated by phosphorylation, and a further increase in STAT3 phosphorylation occurs during reperfusion[24]. A key pathologic event in cardiac ischemia reperfusion injury is mitochondrial energetic dysfunction, and several studies have attributed this to complex I (CxI) inhibition [25]. A previous study have reported that ischemia stimulates the de novo import of mitochondrial STAT3, where it regulates complexes I and II activities [26]. However, this team used STAT3 with a mitochondrial localized sequence (MLS). Moving from these finding reported by other teams, we aimed to determine if ischemia-reperfusion might induce the mitochondrial translocation of STAT3. C57bl6J mice were subjected to $45 \mathrm{~min}$ of ischemia followed by $15 \mathrm{~min}$ of reperfusion $(\mathrm{n}=5)$. Samples were harvested from the area at risk, proteins were extracted and analyzed by western blotting. PS727-STAT3 and total STAT3 were checked along with the cytosolic proteins GAPDH and tubulin. The mitochondrial proteins NDUFB6 (complex I subunit), GRIM-19 andcytochrome C were also detected. A strong total STAT3 signal was detected in the cytosolic fraction and faint bands could be seen in pure mitochondrial fractions (Fig. 1C). However, faint bands could also be detected for the cytosolic freely diffusing GAPDH, while no signal could be found for the cytosolic and polymeric tubulin. Altogether, these results suggested that the faint total STAT3 and GAPDH signals most probably came from contamination of mitochondrial fractions by leakage of cytosolic proteins. Surprisingly, by means of a second batch of anti PS727-STAT3 (b2), we detected a strong band for PS727-STAT3 in the pure mitochondrial fractions exclusively, under both stress and control conditions (Fig. S2). Since the presence of PS727-STAT3 in control mitochondria contradicts what was previously observed in the three different organs experiment, and since PS727-STAT3 is part of the total STAT3 (i.e. total STAT3 must be detected where PS727-STAT3 is detected), we headed toward investigating the presence of such a band through validating the specificity of the antibodies used in these experiments. For this validation, STAT3-KO MEFs were used. The second batch of PS727-STAT3 antibody 
(PS727(b2)-STAT3) detected a dimer in control and a single band in KO cells. This showed that this batch was STAT3 unspecific. The first batch of PS727-STAT3 antibody (PS727-STAT3 b1), PY705-STAT3, and total STAT3 all detected a single band in control cells only $\underline{(n=3)}$ (Fig.1 D). Moreover, in order to ensure the specificity of our antibody beyond just the molecular weight, the complete gel analysis on the whole cardiac cell proteome was performed. The results showed that the total STAT3 antibody had one prominent band at the appropriate STAT3 size, ensuring specificity. However, the complete gel analysis using PS727(b2)STAT3 antibody showed several non-specific bands, which ensures non-specificity (Fig. S2 B and C). Furthermore, the mass spectrometry analysis of the band representing the appropriate STAT3 size showed that STAT3 was identified with 20 unique peptides representing $38 \%$ of sequence coverage. In all the following experiments, only the specific PS727(b1)-STAT3 antibody, which was also validated on serine phosphatase-treated protein extracts (Fig.S1), was used.

\subsection{STAT3 is transversely distributed in cardiomyocytes according to the architecture of T-} tubules

Following the detection of STAT3 by western blotting, we sought to study its localization and distribution pattern in cardiomyocytes (CMs) under various experimental conditions. Our results show that total STAT3 (t-STAT3) is arranged according to a transversal pattern similar to that of ryanodine receptors (RyRs), which lie within regularly spaced transverse striations corresponding to the positions of the T-tubules (Fig. 2A). In addition to the co-labeling of RyR and total STAT3 in CMs, we co-labeled total STAT3 and PS727-STAT3 with the mitochondrial proteins GRIM-19 and TOM22, respectively (Fig. 2 B and C). Our results suggest that STAT3 follows a pattern perpendicular to that of the mitochondrial proteins patterns, with the former being transversely dispersed in the cell, while the latter two are longitudinally dispersed following the normal cellular mitochondrial distribution pattern. The spatial cross-correlation of the fluorescent signals pattern was analyzed (Fig. 3A and 3D) and no cross-correlation could be found between STAT3 signal and mitochondrial signals.

We then studied if hypoxia-reoxygenation stress conditions could modulate the distribution of STAT3 and induce its mitochondrial translocation. CM underwent 90 minutes of hypoxia followed by 60 minutes of reoxygenation. Neither hypoxia (Fig. 3B) nor hypoxia-reoxygenation (Fig. 3C) induced a visible mitochondrial translocation of STAT3. Total STAT3 continued to follow a transversal distribution pattern in the cell and no cross-correlation patterns could be detected between STAT3 and GRIM19 (Fig 3 B and C). 3D images, collected following a Z-stacking, ensured the absence of co-localization of the two proteins (Fig. 3E) although STAT3 signal resided in structures close to mitochondria under such stress conditions. The specificity of antibodies used in the immuno-fluorescence experiments was also pre-validated on STAT3EYFP-transfected cells (Fig. S3).

Finally, we assessed whether STAT3 overexpression could reveal the existence of a mitochondrial transportation mechanism. We thus overexpressed STAT3-EYFP and GRIM19 in H9C2 cardiomyoblasts, and we analyzed the localization of both proteins. A low intensity co-localization could be assumed as 
reported by the faint yellow color displayed by some of the mitochondria, as depicted in the supplementary material (Fig. S4). To validate this eye-viewed co-localization, we used the classical line-intensity profile analysis of STAT3 and GRIM19 signals. At the first glance, a strong over-lap between the two fluorescent signals could be observed at the level of four different mitochondria (four peaks). With a more careful analysis, we observed that, first, the peaks of fluorescence intensity of the 2 wavelengths were slightly shifted for profiles 2 and 3. Taken into account the diffraction limit of optical systems, this suggested that STAT3 signal could have been outside the mitochondria. Second, the $4^{\text {th }}$ and $5^{\text {th }}$ peaks of green fluorescence reporting STAT3 signal showed a similar shape and peak intensity, whereas the $4^{\text {th }}$ peak co-localized with a mitochondria while the $5^{\text {th }}$ was not. This could have been explained by a random co-localization between STAT3 hotspots and mitochondria. Since this line profile analysis does not report a 2D spatial colocalization, we performed the spatial cross-correlation of the fluorescent signals (Fig. S4B). Strikingly, clear and vivid donut-shaped cross-correlation patterns, around the mitochondria, were observed. This what reports that the highest cross-correlation of the two fluorescent signals was found at the mitochondrial circumference rather than inside them. This presents a tipping point toward the probability that STAT3, even when over-expressed, might most-probably be located outside or around the mitochondrial rather than inside the mitochondria themselves.

The fact that we could not detect any clear STAT3 signal in mitochondria, whatever methods we used, does not guarantee that no STAT3 is expressed in mitochondria, but rather that its concentration is below the sensitivity of both Western-blot and immunofluorescence. Our results are in line with those of Phillips et al. who detected very small amount of STAT3 by mass-spectrometry in cardiac cells, at a concentration unlikely to be involved in a biological function in mitochondria[27].

\subsection{The inhibition of STAT3 does not modify mitochondrial function}

The mitochondrial oxygen consumption and calcium retention capacity were measured following pharmacological inhibition of STAT3 by Stattic. Boengler et al[11] originally demonstrated a strong inhibition of complex I and II activities following administration of $100 \mu \mathrm{M}$ Stattic. Literature reports indicate that Stattic can be toxic as previously observed in cancer cells [28]. A dose-dependent assessment of this inhibitor $(10-100 \mu \mathrm{M})$ showed that a $1 \mathrm{~h}$ treatment with 50 and $100 \mu \mathrm{M}$ of Stattic significantly decreased PY705-STAT3 level (>50\%), without any significant effect on PS727-STAT3 level (Fig.4 A and B). However, a $2 \mathrm{~h}$ Stattic treatment induced protein degradation at a $100 \mu \mathrm{M}$ dose (Fig. 4C). Subsequently, a dose of $50 \mu \mathrm{M}$ Stattic, with a $1 \mathrm{~h}$ treatment, was used for further mitochondrial experiments.

The ADP-stimulated oxygen consumption of complexes I, II and IV was recorded following the incubation of crude mitochondria with DMSO (vehicle) or Stattic $(50 \mu \mathrm{M})$ for $1 \mathrm{~h}$ at $4^{\circ} \mathrm{C}$ (with agitation). Our results showed no significant effect for Stattic on complex activities under ADP-stimulated conditions ( $\mathrm{n}=3$ ) (Fig.4 D). The oxygen consumption of complexes I, II, and IV were $35.2 \pm 4.20,25.2 \pm 3.41$ and $80.63 \pm 12.3$ nmole $\mathrm{O}_{2} / \mathrm{min} / \mathrm{mg}$ protein, respectively, following DMSO treatment. These levels slightly and nonsignificantly shifted toward $34.2 \pm 5.24,23.9 \pm 2.57$ and $73.9 \pm 7$ nmole $\mathrm{O}_{2} / \mathrm{min} / \mathrm{mg}$ protein, respectively, following Stattic treatment $(n=3)$. 
Under the same treatment conditions, the calcium retention capacity (CRC) was measured in the presence of glutamate/malate and succinate as substrates for complexes I and II, respectively. ADP and $\mathrm{MgCl}_{2}$ were added for mimicking normal physiological conditions and ensuring optimal mitochondrial respiration. The results show that the CRC was, unexpectedly, increased from $666 \pm 68 \mathrm{nmole}$ calcium/mg protein to $760 \pm$ 86 nmole calcium/mg protein (13\%) in the presence of Stattic (n=3) (Fig.4 E). 


\section{DISCUSSION}

In the present study, taking into account both sensitivity and specificity of the methods used, we could not detect significant levels of STAT3 proteins in mouse heart mitochondria under both baseline conditions and after prolonged ischemia-reperfusion. However, we report a novel prominent distribution of STAT3 along the architecture of T-tubules. We discuss the potential role of STAT3 in the regulation of mitochondrial permeability transition and more largely in cardioprotection

\subsection{STAT3 in heart mitochondria}

In the original study revealing the localization of STAT3 in heart mitochondria, Wegrzyn al. [7] proposed that about $10 \%$ of STAT3 proteins were expressed within mouse heart mitochondria. In the same study, they reported a high concentration of PS727-STAT3 in heart mitochondria. Unfortunately, we could not exactly compare our experiments since the original antibodies which were used are no longer provided by the manufacturers and have been substituted with "more specific" ones as it is indicated on the manufacturer website (Santa Cruz). In the present study, we could not detect 10\% of STAT3 being expressed in mitochondria whatever the anti-STAT3 antibody used (3 different validated antibodies). In a more recent study performed on mouse heart mitochondria as well, the authors also failed to detect PS727-STAT3 in mitochondria [12]. Rainer et al. showed that STAT3 was expressed in wild type heart mitochondria; however, a faint pair of bands was also detected in the mitochondria fraction of STAT3-KO [13]. Although a decrease in intensity can be seen, the fact that bands unrelated to STAT3 can be detected in extract of KO STAT3 cells question the specificity of the antibody. In our experiments with the unspecific S727(b2)STAT3, we detected bands of low intensity of the expected size for STAT3 in STAT3 KO MEFs cells. This result was correlated with an opposite detection of signal in mitochondria vs. cytosolic fractions. Since we used 3 different antibodies validated against KO STAT3 extracts and showing a similar detection in cytosolic but not in mitochondrial fractions, we concluded the non-specificity of the antibody S727(b2)-STAT3. Our results indicate that one should avoid using antibodies that detect bands at the expected size of the candidate protein in its KO models. Following cell fractionation, one cannot guarantee the absolute purity of the fractions, hence only strong signals should be considered as acceptable. Noteworthy, the reference to "pure" mitochondrial fraction instead of enriched mitochondria fraction has maybe become misleading. This technical limitation strongly applies to proteins known to be expressed in ER or mitochondria associated membranes (MAMs), like STAT3 [16], since there is no guarantee that $100 \%$ of these membranes have been retrieved from the "pure" mitochondrial fraction. This might explain why we detected a strong STAT3 signal in crude mitochondrial extracts and virtually no or a non-consistent signal in pure mitochondria. This pattern is indeed the one often observed in several published studies.

The specificity of such detection is usually addressed by labeling compartment-specific proteins in order to show the clear cell compartment discrimination between the different fractions, taking into account a comparable (long enough for weak signals) exposure time of the membrane for all protein markers, together with an appropriate choice of these markers. STAT3 is a soluble protein which is freely diffusing by 
Brownian motion in its media, whether it is cytoplasm, nucleoplasm or mitochondrial matrix. Authors historically used tubulin, a polymer anchored to membrane, as marker of cytosol; VDAC, a transmembrane protein, as marker of the outer mitochondrial membrane; and the sub-unit of the complex I, like GRIM19, as marker of the mitochondrial matrix. Although these markers are good to assess the contamination of fractions by membranes of other compartments, they are not suitable to assess contamination by protein diffusion that would occur when membranes are damaged. Since GRIM19 is a soluble protein, and because only a fraction of GRIM19 proteins is bound to the holoenzyme of complex I, we can consider it as a good marker for mitochondrial matrix. However, we claim here that tubulin is not the best marker to assess for contamination by cytosolic proteins, in such kind of experiments, and should be substituted by another protein such as GAPDH. As shown in (Fig. 1C), tubulin was not detected in the mitochondrial fraction while GAPDH showed a weak band. Accordingly, although we indeed detected a weak STAT3 signal in the mitochondrial fraction, we could not conclude whether it was contamination or a specific localization. In this context too, the weak STAT3 band observed in the pure brain mitochondrial fraction (Fig. 1B) could be due to contamination since a similar signal is observed for both GAPDH and tubulin.

While western blots do not conserve the original spatial information and assesses a cell population, immunofluorescence can be analyzed at the single cell level and provides information as to spatial localization by cross-correlation of signals labelling the proteins of interest and the compartments of interest. However, similar to western blotting, immunofluorescence relies on specificity and sensitivity of the antibodies. Using immunofluorescence we showed a good spatial cross-correlation between STAT3 signal and the ryanodine receptor localized in the junctional SR but no strong cross-correlation between STAT3 and mitochondria. Assuming that near 10\% of STAT3 is expressed in mitochondria, we would have expected to detect a strong spatial cross-correlation between STAT3 and a marker of mitochondria.

We questioned the localization of STAT3 in the mitochondria of cell lines. An extensive literature supports the fact that STAT3 can be translocated to the mitochondria in different cell lines, particularly upon a strong expression in cytosol. According to the literature, experiments targeting STAT3 to the mitochondria by means of a mitochondria-localization site [12] or incubating mitochondria in the presence of a high concentration of STAT3 [14] induced a strong translocation of STAT3 into the mitochondria. An import mechanism of STAT3 to the mitochondria does exist, even though it likely requires either STAT3 overexpression or dysregulation, which could occur in cancer cells. In this study, we reported that the overexpression of STAT3-EYFP in H9C2 cardiomyoblasts-derived cell line was correlated with a significant level of fluorescence in the mitochondria, what supports the fact that a fraction of STAT3 may translocate into the mitochondria under specific conditions. However, our cross-correlation analysis also clearly demonstrated that the major STAT3 signal was actually observed all around the mitochondria not inside them. Given the dotted pattern of STAT3 signal, it is likely that a proportion of STAT3 proteins are bound to structures like membranes rather than freely diffusing in the cytosol. This suggests that a high proportion of STAT3 may be bound to ER membrane surroundings the mitochondria. This hypothesis is supported by a recent study [16] and could explain why a strong STAT3 signal is detected in the crude mitochondrial fraction which includes a large proportion of ER membranes. It could also explain why a STAT3 
contamination could be observed in "pure" mitochondrial fraction, since it is difficult to guarantee $100 \%$ removal of ER membranes.

Several studies reported that upon induction (including ischemia-reperfusion), STAT3 translocates from cytosol to nucleus or to mitochondria. On our side, we failed to detect STAT3 in mitochondria after ischemia-reperfusion, with both Western-blot and immunofluorescence, in both in vivo and in vitro models while, conversely, a strong signal was observed in cells nuclei.

Altogether, our results do not support a role for STAT3 in mouse cardiac mitochondria [12].

\subsection{Specificity, sensitivity, and toxicity of Stattic}

Stattic is a specific inhibitor of STAT3 [29] and has been used in numerous studies. We observed that in some studies [13], high concentration of Stattic $(>100 \mu \mathrm{M})$ were used regardless its IC50 for STAT3 phosphorylation $\left(20 \mu \mathrm{M}\right.$ for $1 \mathrm{hr}$ incubation at $\left.30^{\circ} \mathrm{C}\right)$ and for cell death induction $(20 \mu \mathrm{M})$ [29]. Noteworthy, in the original article showing Stattic specificity, Stattic inhibited STAT1 at 40\% with an IC50 of $10 \mu \mathrm{M}$ at $30^{\circ} \mathrm{C}$. In a recent study, authors confirmed that $10 \mu \mathrm{M}$ Stattic inhibited both STAT1 and STAT3 phosphorylation activity [28]. In this latter study, a prolonged $20 \mu \mathrm{M}$ Stattic treatment was found to be toxic and induced cell death. In our hands, a $100 \mu \mathrm{M}$ Stattic treatment for $2 \mathrm{~h}$ induced STAT3 and tubulin degradation confirming that high Stattic concentration could trigger toxic effects non-related to its effect on STAT3 (Fig. 4C). We could not detect any inhibition of mitochondrial respiration when we treated isolated mitochondria with $50 \mu \mathrm{M}$ for $1 \mathrm{~h}$ at $4^{\circ} \mathrm{C}$ (this temperature was used to preserve mitochondria during incubation). Boengler et al found a 50\% decrease in complex I activity when incubating heart mitochondria with $200 \mu \mathrm{M}$ Stattic for $1 \mathrm{~h}$ at $4^{\circ} \mathrm{C}$ [13]. Although the temperature of incubation is slowing down the reaction, Stattic can be considered as being in saturating condition since majority of cellular STAT3 is absent in crude mitochondrial fraction. Therefore, it is very unlikely that we could not observe an inhibitory effect of $50 \mu \mathrm{M}$ Stattic if STAT3 is expressed in mitochondria and is regulating complex I activity. Conversely, the use of $200 \mu \mathrm{M}$ Stattic could have induced: i) protein degradation and subsequent toxic effect downregulating complex I activity, and ii) inhibition of STAT1 as it was detected in heart mitochondria [13]. Besides, it should be kept in mind that respiration and CRC experiments are usually carried on crude mitochondrial fractions, which incorporates a lot of STAT3 protein outside of the mitochondria. In case a direct effect on STAT3 inhibition would be observed, such as the small increase in CRC we observed, it should not be claimed that it is more likely related to an effect on STAT3 from the inside of mitochondria rather than from the outside of the mitochondria. In conclusion, both data of the literature and our results suggest a careful control of the non-specific effect of Stattic, particularly in experiments with cell fractions which may include no or low STAT3 levels.

\subsection{The STAT3 KO phenotype}

Previous reports suggest that mitochondrial respiration is down-regulated in cardiomyocytes of STAT3 KO mice and in STAT3 KO cell lines [7] [30]. Since STAT3 is primarily a transcription factor and is known to regulate genes involved in metabolism and mitochondrial activity :[31], [32], one should not exclude a 
canonical genomic explanation to the down-regulation of respiration in heart mitochondria of STAT3 KO mice. In addition, sharing an apparent similar phenotype between in vivo cardiomyocytes and in vitro cell lines could not mandatorily be recapitulated by a common mechanism. Indeed, since we could not detect STAT3 in heart mitochondria but could see STAT3-EYFP around mitochondria of H9C2, it is unlikely that the effect of STAT3 on respiration would be caused by a common mechanism.

In conclusion, our results show a novel vivid distribution for STAT3 along the T-tubules of cardiomyocytes and in the nucleus, under different conditions. Moreover, we neither find a significant amount of STAT3 in mouse heart mitochondria, nor detect a direct involvement for it in the regulation of mitochondrial activities under physiologic conditions. Although we agree that under non-physiological conditions, such as STAT3 overexpression or MLS-tagged STAT3, STAT3 could be translocated to mitochondria, we speculate that this mechanism could mainly be relevant to cell lines already showing a strong expression of STAT3. Alternatively, our localization and functional experiments support the fact that STAT3 is localized around the mitochondria in a cardiomyoblasts cell line, and it may indeed support an indirect effect on their activity. 


\section{AUTHOR CONTRIBUTIONS}

Conceived experiments: ZH, SB, MK, GB.

Performed experiments: ZH, SB, NGB, MP, BP, LA.

Wrote the manuscript: $\mathrm{ZH}, \mathrm{MO}$ and GB.

Supervised the study: MK, GB.

Review the manuscript: MP, GWB, ECS, MO.

The following are the supplementary data related to this article.

Figure S1: Specificity of the PS727(b1)-STAT3 antibody

Western blot analysis showing the effect of a serine-phosphatase treatment on the level of phosphorylation of Y705 and S727 STAT3. Tubulin reports the protein loads in each well. Three independent experiments were carried out on different mouse hearts.

Figure S2: Lack of specificity of PS727(b2)-STAT3 antibody in mitochondrial and complete cardiac cell proteomic fractions

(A) Western blot analysis of PS727(b2)-STAT3, total STAT3, tubulin, GAPDH, Cytochrome C and GRIM-19 in pure mitochondrial extracts from the areas at risk of mice hearts subjected to 45 minutes of ischemia followed by 15 minutes of reperfusion or from control mice. Note that while PS727(b2)-STAT3 detects a band in the mitochondrial fraction, no total STAT3 can be detected, which is unfathomable. Six independent experiments were carried out on different mouse hearts.

(B) Western blot analysis of PS727(b2)-STAT3 in total cardiomyocyte proteomic sample.

(C) Western blot analysis of Total STAT3 in total cardiomyocyte proteomic sample

Figure S3: Specificity of the STAT3 antibodies in immunofluorescence experiments.

Confocal images of H9C2 cells transfected with STAT3-EYFP plasmids for 48h and then labeled with different antibodies:

I- Specific used antibodies (A) rabbit IgG anti-total STAT3 (tSTAT3), (B) mouse IgG anti- S727 phosphorylation of STAT3 (PS727(b1)-STAT3);

II- Non-specific unused antibodies (C) mouse IgG anti-total STAT3 (mouse tSTAT3) and (D) rabbit IgG anti- S727 phosphorylation of STAT3 (rabbit PS727-STAT3). (E) and (F), Immunofluorescence experiments on mouse isolated cardiomyocytes show the detection of TOM22 and GRIM19 proteins, respectively, in mitochondria and concomitantly to the non-specific fluorescent signal given by mouse $\operatorname{IgG}$ anti-total STAT3 (mouse tSTAT3) and rabbit IgG anti- S727 phosphorylation of STAT3 (rabbit PS727STAT3), respectively. Three independent experiments were carried out on different mouse hearts.

Figure S4: STAT3 and GRIM19 co-localize at the mitochondrial circumference in H9C2 cardiomyoblasts.

(A) Confocal images of H9C2 cardiomyoblasts co-transfected with STAT3-EYFP (green signal) and mCherry GRIM19 (red signal) plasmids for 48h (left panel), along with the line intensity profiler (middle right panel) and plot showing intensity profiles of both signals in four different randomly chosen mitochondria (right panel). Scale bar $10 \mu \mathrm{m}$ 
(B) Map of the spatial cross-correlation intensity (CCI) of the two fluorescent signals (left panel) and a representation of the correlation pattern of distribution (red pattern) in a randomly chosen zoomed-in area (right panel), showing a donut-shaped co-localization pattern around mitochondria rather than inside them. Scale bar $1 \mu \mathrm{m}$

\section{AKNOWLEGDMENTS}

This study benefits from grants offered by INSERM, the Lebanese University, and The Cedar program of Campus France (\#37303QA 2017-2018).

This work was supported by the IHU OPeRa (ANR-10-IBHU-004) within the program "Investissements d'Avenir" operated by the French National Research Agency (ANR).

Zeina Harhous was supported by the "Association de Specialisation et d'orientation Scientifique".

Sally Badawi was supported by the Council for Scientific Research and Lebanese University fellowship (CNRS-L) program and the Eiffel scholarship program of excellence of Campus France.

$\underline{\text { We acknowledge the financial support from ITMO Cancer AVIESAN (Alliance Nationale pour les Sciences }}$ de la Vie et de la Santé, National Alliance for Life Sciences and Health) within the framework of the cancer plan for Orbitrap mass spectrometer founding.

We also thank Adeline Page for performing the mass spectrometry analysis (Protein Science Facility, SFR BioSciences CNRS UMS3444, Inserm US8, UCBL, ENS de Lyon, 50 Avenue Tony Garnier, 69007 Lyon, France). 


\section{REFERENCES}

[1] K. Boengler, D. Hilfiker-Kleiner, H. Drexler, G. Heusch, et R. Schulz, "The myocardial JAK/STAT pathway: From protection to failure », Pharmacol. Ther., vol. 120, $\mathrm{n}^{\mathrm{0}} 2$, p. 172-185, nov. 2008.

[2] X. Cheng, C. Peuckert, et S. Wölfl, «Essential role of mitochondrial Stat3 in p38MAPK mediated apoptosis under oxidative stress », Sci. Rep., vol. 7, nov. 2017.

[3] L. M. LaFave et R. L. Levine, «JAK2 the future: therapeutic strategies for JAKdependent malignancies », Trends Pharmacol. Sci., vol. 33, nº 11, p. 574-582, nov. 2012.

[4] I. Hazan-Halevy et al., «STAT3 is constitutively phosphorylated on serine 727 residues, binds DNA, and activates transcription in CLL cells », Blood, vol. 115, $\mathrm{n}^{\mathrm{o}}$ 14, p. 28522863, avr. 2010.

[5] N. Sato et al., " Physical and functional interactions between STAT3 and ZIP kinase », Int. Immunol., vol. 17, $\mathrm{n}^{\mathrm{o}}$ 12, p. 1543-1552, déc. 2005.

[6] Z. Wen, Z. Zhong, et J. E. Darnell, « Maximal activation of transcription by statl and stat3 requires both tyrosine and serine phosphorylation », Cell, vol. 82, n 2, p. 241-250, juill. 1995.

[7] J. Wegrzyn et al., «Function of mitochondrial Stat3 in cellular respiration », Science, vol. 323, nº 5915, p. 793-797, févr. 2009.

[8] L. Gomez et al., «Inhibition of mitochondrial permeability transition improves functional recovery and reduces mortality following acute myocardial infarction in mice », Am. J. Physiol.-Heart Circ. Physiol., vol. 293, n 3 3, p. H1654-H1661, sept. 2007.

[9] D. J. Hausenloy, H. L. Maddock, G. F. Baxter, et D. M. Yellon, « Inhibiting mitochondrial permeability transition pore opening: a new paradigm for myocardial preconditioning? », Cardiovasc. Res., vol. 55, nº 3, p. 534-543, août 2002.

[10] S. A. Javadov, S. Clarke, M. Das, E. J. Griffiths, K. H. H. Lim, et A. P. Halestrap, «Ischaemic preconditioning inhibits opening of mitochondrial permeability transition pores in the reperfused rat heart », J. Physiol., vol. 549, n Pt 2, p. 513-524, juin 2003.

[11] K. Boengler, E. Ungefug, G. Heusch, et R. Schulz, «The STAT3 inhibitor stattic impairs cardiomyocyte mitochondrial function through increased reactive oxygen species formation », Curr. Pharm. Des., vol. 19, nº 39, p. 6890-6895, 2013.

[12] K. Szczepanek et al., «Cardioprotective function of mitochondrial-targeted and transcriptionally inactive STAT3 against ischemia and reperfusion injury », Basic Res. Cardiol., vol. $110, \mathrm{n}^{\mathrm{o}} 6$, p. 53, nov. 2015.

[13] K. Boengler, D. Hilfiker-Kleiner, G. Heusch, et R. Schulz, « Inhibition of permeability transition pore opening by mitochondrial STAT3 and its role in myocardial ischemia/reperfusion », 
Basic Res. Cardiol., vol. 105, nº 6, p. 771-785, nov. 2010.

[14] P. Tammineni, C. Anugula, F. Mohammed, M. Anjaneyulu, A. C. Larner, et N. B. V. Sepuri, «The Import of the Transcription Factor STAT3 into Mitochondria Depends on GRIM-19, a Component of the Electron Transport Chain », J. Biol. Chem., vol. 288, n ${ }^{\text {7 }}$, p. 4723-4732, févr. 2013.

[15] Q. Zhang et al., «Mitochondrial localized Stat3 promotes breast cancer growth via phosphorylation of serine 727 », J. Biol. Chem., vol. 288, n 43, p. 31280-31288, oct. 2013.

[16] L. Avalle et al., «STAT3 localizes to the ER, acting as a gatekeeper for ERmitochondrion Ca2+ fluxes and apoptotic responses », Cell Death Differ., juill. 2018.

[17] L. Avalle et V. Poli, « Nucleus, Mitochondrion, or Reticulum? STAT3 à La Carte », Int. J. Mol. Sci., vol. 19, n ${ }^{\circ}$ 9, p. 2820, sept. 2018.

[18] R. Harisseh et al., « Unacylated ghrelin analog prevents myocardial reperfusion injury independently of permeability transition pore », Basic Res. Cardiol., vol. 112, n 1, p. 4, déc. 2016.

[19] A. Gharib et al., "Opposite and tissue-specific effects of coenzyme Q2 on mPTP opening and ROS production between heart and liver mitochondria: Role of complex I », J. Mol. Cell. Cardiol., vol. 52, n ${ }^{\circ}$ 5, p. 1091-1095, mai 2012.

[20] M. R. Wieckowski, C. Giorgi, M. Lebiedzinska, J. Duszynski, et P. Pinton, «Isolation of mitochondria-associated membranes and mitochondria from animal tissues and cells », Nat. Protoc., vol. 4, n ${ }^{\text {o }} 11$, p. 1582-1590, nov. 2009.

[21] D. De Paulis et al., «Cyclosporine A at reperfusion fails to reduce infarct size in the in vivo rat heart », Basic Res. Cardiol., vol. 108, n 5, p. 379, août 2013.

[22] T. D. O’Connell, M. C. Rodrigo, et P. C. Simpson, «Isolation and Culture of Adult Mouse Cardiac Myocytes », in Cardiovascular Proteomics, vol. 357, New Jersey: Humana Press, 2006, p. 271-296.

[23] G. Bidaux et al., «FRET Image Correlation Spectroscopy Reveals RNAPIIIndependent P-TEFb Recruitment on Chromatin », Biophys. J., vol. 114, n ${ }^{0}$ 3, p. 522-533, févr. 2018.

[24] K. Boengler, « Ischemia/reperfusion injury: The benefit of having STAT3 in the heart », J. Mol. Cell. Cardiol., vol. 50, nº 4, p. 587-588, avr. 2011.

[25] A. J. Tompkins, L. S. Burwell, S. B. Digerness, C. Zaragoza, W. L. Holman, et P. S. Brookes, «Mitochondrial dysfunction in cardiac ischemia-reperfusion injury: ROS from complex I, without inhibition », Biochim. Biophys. Acta BBA - Mol. Basis Dis., vol. 1762, nº 2, p. 223-231, févr. 2006.

[26] K. Szczepanek et al., «Mitochondrial-targeted Signal transducer and activator of 
transcription 3 (STAT3) protects against ischemia-induced changes in the electron transport chain and the generation of reactive oxygen species », J. Biol. Chem., vol. 286, n ${ }^{\circ}$ 34, p. 29610-29620, août 2011.

[27] D. Phillips et al., «Stoichiometry of STAT3 and mitochondrial proteins: Implications for the regulation of oxidative phosphorylation by protein-protein interactions », J. Biol. Chem., vol. 285, nº 31, p. 23532-23536, juill. 2010.

[28] I. Sanseverino, C. Purificato, M. C. Gauzzi, et S. Gessani, «Revisiting the specificity of small molecule inhibitors: the example of stattic in dendritic cells », Chem. Biol., vol. 19, $\mathrm{n}^{\mathrm{o}}$ 10, p. 1213-1214; author reply 1215-1216, oct. 2012.

[29] J. Schust, B. Sperl, A. Hollis, T. U. Mayer, et T. Berg, « Stattic: a small-molecule inhibitor of STAT3 activation and dimerization », Chem. Biol., vol. 13, n 11, p. 1235-1242, nov. 2006.

[30] R. Yang et al., «Mitochondrial Ca2+ and membrane potential, an alternative pathway for Interleukin 6 to regulate CD4 cell effector function », eLife, vol. 4, mai 2015.

[31] V. Poli et A. Camporeale, «STAT3-Mediated Metabolic Reprograming in Cellular Transformation and Implications for Drug Resistance », Front. Oncol., vol. 5, juin 2015.

[32] M. Demaria, A. Camporeale, et V. Poli, «STAT3 and metabolism: How many ways to use a single molecule?: STAT3 and Metabolism », Int. J. Cancer, vol. 135, n 9, p. 1997-2003, nov. 2014. 


\section{FIGURE LEGENDS}

Figure 1. Cytosolic and mitochondrial fractions show the absence of STAT3 in pure mitochondria of different organs.

Western blots showing the detection of Y705- and S727-phosphorylated and total STAT3 forms; the cytosolic proteins tubulin and GAPDH; and the mitochondrial proteins Tom20, GRIM19, cytochrome C and NDUFB6 (complex I subunit) in (A) protein extract of crude mitochondria fraction $(40 \mu \mathrm{g}) \underline{(\mathrm{n}=3)}$, (B) pure mitochondrial and cytosolic extracts from hearts, brains, and livers of control C57bl/6J mice (90 $\mu \mathrm{g}$ of protein extract per well) $(\mathrm{n}=3)$, (C) pure mitochondrial extracts from the area-at-risk of mouse heart subjected to 45 minutes of ischemia followed by 15 minutes of reperfusion (30 $\mu \mathrm{g}$ of protein extract per well) $(\mathrm{n}=5)$, (D) verification of the specificity of several STAT3 antibodies in $40 \mu \mathrm{g}$ of total protein extract of wild type ( $w t)$ and STAT3-KO MEFs: PS727-STAT3 (batches one and two: b1 and b2), PY705-STAT3 and total STAT3 $(\mathrm{n}=3)$.

Figure 2. STAT3 is distributed in nucleus and along t-tubules of cardiomyocytes.

Confocal images of mouse isolated cardiomyocytes immuno-labelled with antibodies against (A) a marker of junctional sarcoplasmic reticulum, the ryanodine receptor type II (RyR2) in red and total STAT3 (green), (B) a mitochondrial marker, GRIM-19 (Red), and total STAT3 (Green) or the mitochondrial marker Tom20 (green) and (C) PS727(b1)-STAT3 (Red). Depth of the focal plane: $1 \mu \mathrm{m}$. Three independent experiments were carried out on different mouse hearts. Scale bars: $10 \mu \mathrm{m}$.

Figure 3. Hypoxia and hypoxia-reoxygenation do not induce the mitochondrial translocation of STAT3 in mouse-isolated cardiomyocytes.

Confocal images showing the merging of fluorescence image of total STAT3 (green) and GRIM-19 (red) in mouse isolated cardiomyocytes incubated under (A) normoxia, (B) hypoxia for $90 \mathrm{~min}$ or (C) hypoxia as in (B) followed by reoxygenation for $1 \mathrm{~h}$ (HR). Right images are quantifying the spatial cross-correlation intensity (CCI) of the 2 fluorescent signals. (D) The confocal image and co-localization pattern of total STAT3 (green) and RyR2 (red) in cardiomyocytes under normoxia conditions. (E) A 3D reconstructed projection showing the localization pattern of total STAT3 (green) and GRIM-19 (Red) in cardiomyocytes subjected to HR. Top row of image is showing the 3D rendering from above for merged fluorescence (left), STAT3 fluorescence alone (middle) and GRIM-19 fluorescence alone (right). Bottom row showing the 3D rendering from below (left) and the 3D spatial cross-correlation image. Three independent experiments were carried out on different mouse hearts. Scale bar: $10 \mu \mathrm{m}$.

Figure 4: Pharmacological inhibition of STAT3 by Stattic has no effect on ADP-stimulated mitochondrial oxygen consumption but slightly increases calcium retention capacity.

(A) Western-blots showing the dose-effect of a one hour- or (C) two hours- treatment with the STAT3 inhibitor, Stattic, on the phosphorylation of STAT3 in mouse isolated adult cardiomyocytes. (B) Phosphorylation of Y705 and S727 residues of STAT3 was quantified and divided by the amount of total STAT3 protein. STAT3 ratios for one hour Stattic-treated cells were normalized by DMSO treated condition. (D) Oxygen consumption rate by complexes I, II and IV (state 3) measured in isolated mitochondria of mouse cardiomyocytes, incubated with either DMSO or $50 \mu \mathrm{M}$ Stattic for $1 \mathrm{~h}$ at $4^{\circ} \mathrm{C}$. (E) Calcium retention 
capacity measurement for mitochondria treated with DMSO or $50 \mu \mathrm{M}$ Stattic for $1 \mathrm{~h}$ at $4^{\circ} \mathrm{C}$. Three independent experiments were carried out on different mouse hearts. 


\section{Highlights}

Our Current study, Critical Appraisal of Mitochondrial STAT3 in Adult Cardiomyocytes, highlights the following findings:

- The study of the spatial distribution of STAT3 signal, in adult mouse cardiomyocytes, revealed that STAT3 is transversely distributed along the T-tubules and in nucleus but not in the mitochondria.

- $\quad$ STAT3 is exclusively detected in crude but not pure mitochondria of heart, brain and liver under basal and ischemia/reperfusion conditions.

- Underlining of technical methods, complemental to cell fractionation, to evaluate STAT3 roles in cardiac cells under different conditions.

- The pharmacologic inhibition of STAT3 has no significant effect on the mitochondrial respiration, but it induces a small significant increase in the calcium retention capacity. 